SLEEP DISORDERED BREATHING

\title{
Continuous positive airway pressure reduces daytime sleepiness in mild to moderate obstructive sleep apnoea: a meta-analysis
}

\author{
N S Marshall, M Barnes, N Travier, A J Campbell, R J Pierce, R D McEvoy, A M Neill, \\ P H Gander
}

See end of article for authors' affiliations

Correspondence to Dr N S Marshall, Sleep and Circadian Research Group, Woolcock Institute of Medical Research, P O Box M77, Missenden Road, NŚW 2050

Australia; nmarshall@ woolcock.org.au

Received 3 August 2005 Accepted 24 January 2006 Published Online First 7 February 2006
Background: Obstructive sleep apnoea syndrome (OSAS) affects an estimated $2-4 \%$ of the middle aged population. Meta-analyses of randomised controlled trials have shown that the severe presentation of the syndrome (apnoea hypopnoea index (AHI) $>30 /$ hour) is effectively treated with continuous positive airway pressure (CPAP). Until recently there have been insufficient data to determine whether CPAP improves sleepiness in the larger subgroup with mild to moderate OSAS (AHI 5-30/hour).

Methods: A systematic search of Medline and a hand search identified seven randomised controlled trials where CPAP was compared with either a placebo or with conservative management in the treatment of mild to moderate OSAS (AHI 5-30/hour). All trials used the Epworth Sleepiness Scale (ESS), four used the Multiple Sleep Latency Test (MSLT), and three used the Maintenance of Wakefulness Test (MWT) to measure sleepiness.

Results: Meta-analyses indicated that CPAP significantly reduced subjective daytime sleepiness (ESS) by 1.2 points $(95 \% \mathrm{Cl} 0.5$ to $1.9, \mathrm{p}=0.001)$, improved objective daytime wakefulness (MWT) by 2.1 minutes (95\% Cl 0.5 to 3.7, $\mathrm{p}=0.011$ ), but did not affect objective daytime sleepiness (MSLT, mean benefit -0.2 minutes, $95 \% \mathrm{Cl}-1.0$ to $0.6, \mathrm{p}=0.6$ ). The two significant effects were small (effect size $<0.30$ ). Conclusions: CPAP elicits small improvements in subjective sleepiness and objective wakefulness in people with mild to moderate OSAS. However, the effects on sleepiness are of limited clinical significance.
O bstructive sleep apnoea syndrome (OSAS) is a common sleep breathing disorder characterised by repetitive collapse or narrowing of the airway resulting in intermittent blood oxygen desaturations and fragmented sleep. OSAS, coupled with daytime sleepiness, affects at least $2-4 \%$ of the middle aged population. ${ }^{1}$ Sleepiness is a common presenting symptom of OSAS and may be measured by both subjective ${ }^{2}$ and objective methods. ${ }^{3}$ Other presenting symptoms include loud sonorous snoring, cognitive confusion, poor executive functioning, impaired quality of life, morning headaches, and hypertension. It is well established that continuous positive airway pressure $(\mathrm{CPAP})^{5}$ effectively reduces obstructive sleep disordered breathing to levels regarded as normal. The most recent meta-analysis of published randomised controlled trials has also shown that CPAP effectively reduces both subjective and objective daytime sleepiness in people with severe OSAS. ${ }^{6}$ However, most people with the condition have mild to moderate severity OSA, which is defined by an apnoea hypopnoea index (AHI) of 5-30/hour plus daytime sleepiness. ${ }^{78}$ From the four randomised controlled trials ${ }^{9-12}$ available in 2001, Patel and colleagues ${ }^{6}$ were unable to conclude that CPAP was effective in reducing daytime sleepiness in this patient population. However, this sub-analysis included one additional study that specifically investigated the use of CPAP in patients with AHI >30/hour but no daytime sleepiness. ${ }^{13}$ That study addressed a separate question regarding treatment of severe sleep disordered breathing without marked daytime sleepiness $(\mathrm{AHI}>30)$ and does not bear directly on the effectiveness of CPAP in mild to moderate OSAS. Since the meta-analysis by Patel and colleagues, ${ }^{6}$ we have published three additional randomised controlled trials examining the effectiveness of CPAP treatment for patients with mild to moderate OSAS. ${ }^{14-16}$ Here we report on a new systematic search and meta-analysis to quantify the effect of CPAP on daytime sleepiness in people with mild to moderate OSAS.

\section{METHODS}

Evidence search

A systematic review of the current literature was undertaken using Medline publications from 1 January 1994 to 31 December 2004. Keywords used to identify randomised controlled trials of CPAP in the treatment of mild to moderate OSAS published in English were (apnoea.af or apnea.af or hypopnoea.af or hypopnea.af) and (CPAP.af or continuous positive airway pressure.af or positive airways pressure.af or positive pressure.af) and (randomized controlled trial.pt or .ti or clinical trial.pt or .ti). Suffixes indicated search fields used in MEDLINE (. af $=$ all fields, $. p t=$ publication type, $. t i=$ title $)$. The authors' personal knowledge, previous randomised trials, meta-analyses and reviews (including previous Cochrane reviews) were used as additional bibliographic sources.

\section{Selection of published studies}

Two investigators (NSM and MB) independently applied the following selection rules to the studies identified in the systematic search. Only studies that were randomised controlled trials with treatment arms of at least 1 week of manually titrated CPAP therapy undertaken on adult patients

Abbreviations: $\mathrm{AHI}$, apnoea hypnoea index; $\mathrm{BMI}$, body mass index; CPAP, continuous positive airway pressure; ESS, Epworth Sleepiness Scale; MSLT, Multiple Sleep Latency Test; MWT, Maintenance of Wakefulness Test; OSAS, obstructive sleep apnoea syndrome 
with OSAS were considered. One of the arms had to be either conservative management or placebo. Conservative management of OSAS usually includes advice about weight loss, sleep hygiene, adoption of non-supine sleep posture, and avoidance of alcohol and sedatives. Acceptable placebos included orally ingested placebo tablets or sham CPAP devices that did not improve overnight indices of sleep disordered breathing compared with baseline polysomnographic reports. Unacceptable comparisons included any device that improved sleep disordered breathing such as suboptimally pressured but partially therapeutic CPAP, or any surgical techniques including sham surgery or sham or ineffective mandibular advancement splints. Studies not exclusively analysing mild to moderate OSAS classification (AHI specified as 5-30/hour) were excluded. To be included, trials had to measure the Epworth Sleepiness Scale (ESS; a measure of trait subjective sleepiness, self-reported likelihood of dozing in common situations ${ }^{2}$ ), and/or the Multiple Sleep Latency Test (MSLT; a measure of state objective sleepiness, propensity to fall asleep ${ }^{3}$ ), and/or the Maintenance of Wakefulness Test (MWT, a measure of state objective wakefulness, the ability to resist sleep ${ }^{4}$ ). The ESS is a pencil and paper measure of the likelihood of falling asleep in eight everyday situations (each scored between 0 and 3) and is expressed as a score between 0 (not sleepy) and 24 (extremely sleepy). The two objective methods quantify daytime sleepiness by electrophysiologically measuring the average sleep latency across a number of nap opportunities in a single day. They mainly differ by the instruction to patients to "go to sleep" (MSLT) or to "stay awake" (MWT).

\section{Data abstraction and trial quality assessment}

Study and patient characteristics retrieved for each trial included: number of patients, placebo type, trial structure, length of follow up, sex balance, mean age, mean body mass index (BMI; $\mathrm{kg} / \mathrm{m}^{2}$ ), mean AHI, method of measuring airflow (thermistor or pressure transducer), dropout rate, mean CPAP use per night, country of origin, and baseline severity of sleepiness measured by the ESS, MWT, and/or MSLT. The primary data analyses required the mean (SE) of the placebo adjusted effects of CPAP on the ESS, MSLT or MWT. The Jadad $\operatorname{score}^{17}$ is a validated metric used to assess the quality of randomised controlled trials. Points are awarded from 0 to 5 for appropriate double blinding, appropriate randomisation, and the clear reporting of trial dropouts and exclusions. Jadad scores can be used in a meta-analysis to assess whether trial quality has affected the size of the treatment effect and to exclude trials of lower quality. Jadad scores were independently calculated by two investigators for all included trials.

\section{Data synthesis and statistical analysis}

The differences in treatment effect between CPAP and control and their standard errors were not always calculable from information in the published papers. However, Patel and colleagues $^{6}$ have recently collected and published this information from the original authors of the four papers published before 2001 .

The mean differences in treatment responses between CPAP and placebo groups and the standard error of that difference from individual studies were meta-analysed (META) using Stata Version 7.0 (Stata Corp, College Station, TX, USA). Pooled estimates of treatment responses were calculated with both fixed and random effects model assumptions. Heterogeneity in treatment effect between trials was tested for using the Q statistic. Meta-regressions (METAREG) were performed to explore the possible sources of any observed heterogeneity in treatment effects. Variables potentially associated with CPAP efficacy (trial and patient

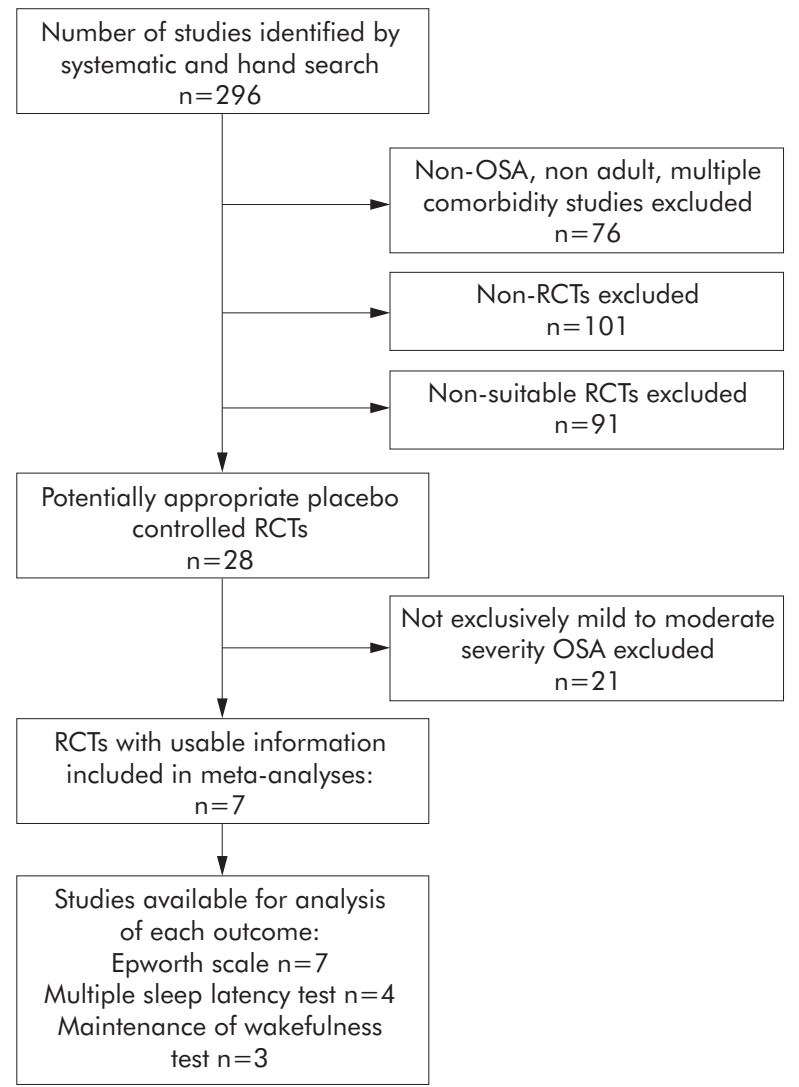

Figure 1 Trial inclusion flow chart.

characteristics) were successively incorporated into a single covariate model to assess their contribution to heterogeneity. Summary estimates were also calculated excluding each study sequentially to measure the influence of individual study effects on the pooled estimate (METAINF). Publication bias was evaluated visually using the funnel plot and statistically (METABIAS) using Egger's and Begg's tests. Effect sizes ${ }^{18}$ were calculated by dividing the mean size of the effect by the baseline standard deviation of a suitable published comparison group of 110 untreated Australian patients with mild to moderate OSA. ${ }^{19}$

\section{RESULTS}

\section{Systematic review}

The systematic search of Medline identified 295 studies of further interest. An additional suitable study published outside the search dates was also included. ${ }^{16}$ The search terms used were not specific to our question. Thus, many of the studies identified were not randomised controlled trials, did not investigate OSAS, or involved paediatric patients or patients with OSAS and other serious co-morbidities. Many unsuitable trials investigated CPAP (or a similar such device) compared with mandibular advancement splints, autotitrating CPAP, bi-level PAP, or surgical procedures. A number of trials used what were described as "placebo CPAP machines". ${ }^{20-25}$ On closer inspection these machines substantially reduced AHI and thus were not placebo devices. Another trial compared positional therapy with CPAP in a group of patients who had largely mild to moderate positional OSAS. ${ }^{26}$ Thus, the positional therapy might be expected to be beneficial to this subgroup and was not a valid placebo. After reviewing the abstracts of the remaining studies, 28 were randomised placebo/conservative management controlled trials of CPAP. Both reviewers independently 
Table 1 Characteristics of included studies

\begin{tabular}{|c|c|c|c|c|c|c|c|}
\hline Reference & No of patients & Control type & $\begin{array}{l}\text { Crossover } \\
\text { study }\end{array}$ & $\begin{array}{l}\text { Treatment } \\
\text { duration (weeks) }\end{array}$ & $\begin{array}{l}\text { Dropout } \\
\text { rate }\end{array}$ & $\begin{array}{l}\text { MSLT or } \\
\text { MWT }\end{array}$ & Jadad score \\
\hline Engleman $^{10}$ & 16 and 9 & Pill & $Y$ & 4 & $11 \%$ & MSLT & 3 \\
\hline Redline ${ }^{12}$ & 97 & $\mathrm{CT}$ & $\mathrm{N}$ & 10.5 & $13 \%$ & MSLT & 3 \\
\hline Engleman ${ }^{9}$ & 34 & Pill & Y & 4 & $8 \%$ & MWT & 3 \\
\hline Monasterio" & 125 & $\mathrm{CT}$ & $N$ & 24 & $12 \%$ & MSLT & 2 \\
\hline Barnes $^{14}$ & 28 & Pill & $Y$ & 8 & $33 \%$ & MSLT & 3 \\
\hline Barnes $^{15}$ & 80 & Pill & $N$ & 12 & $30 \%$ & MWT & 3 \\
\hline Marshall ${ }^{16}$ & 29 & Sham CPAP & $Y$ & 3 & $6 \%$ & MWT & 3 \\
\hline
\end{tabular}

agreed that seven of these investigated mild to moderate OSAS and were suitable for inclusion. Figure 1 shows the trial inclusion flow chart. All seven studies used the ESS, ${ }^{9-12}$ 14-16 four used the MSLT ${ }^{10-1214}$ and the remaining three used the MWT. ${ }^{15} 16$ The characteristics of the included trials are summarised in table 1 , and table 2 summarises the patient characteristics from each of the trials. Five of the seven studies used nasal thermistors ${ }^{1012} 1415$ to measure airflow while the remaining two used nasal pressure ${ }^{11} 16$ in accordance with recent guidelines. ${ }^{8}$ Trials using nasal thermistors might include more severely afflicted OSAS patients as this method is less sensitive to breathing disturbances than nasal pressure measurements.

Six studies scored 3 on the Jadad scale of trial quality. All these had adequate descriptions of trial dropouts and withdrawals in addition to being appropriately randomised. In one study it was unclear why dropouts had occurred. ${ }^{11}$ No studies were truly double blind as CPAP titrations require that at least some of the patients' interactions with sleep staff require those staff being unblinded. This holds even where placebo CPAP devices are used. ${ }^{16}$

\section{Meta-analyses}

After controlling for placebo effects, Epworth scores were significantly improved by CPAP by 1.2 points (95\% CI 0.5 to 1.9, $\mathrm{p}=0.001$, fig 2). The random effects model was used because effects on the ESS were heterogeneous $(\mathrm{Q}=13.1$, $\mathrm{df}=6, \mathrm{p}=0.04)$. Meta-regression indicated that heterogeneity was only related to one of the trial or patient characteristics extracted from the studies (tables 1 and 2 and also thermistor or pressure transducer measurement of SDB). Studies that had lower dropout rates had larger reductions in Epworth scores $(\mathrm{Z}=2.38, \mathrm{p}=0.02)$. Mean compliance with CPAP therapy did not explain the heterogeneity in improvements on the ESS. CPAP still had a small but significantly greater than zero effect when crossover and parallel trials were analysed separately.

CPAP treatment led to an improvement in the MWT of 2.1 minutes (95\% CI 0.5 to $3.7, \mathrm{p}=0.011)$ when using both fixed and random effects methods (fig 3). MWT effects were not heterogeneous $(\mathrm{Q}=1.3, \mathrm{df}=2, \mathrm{p}=0.53)$. Only two of the

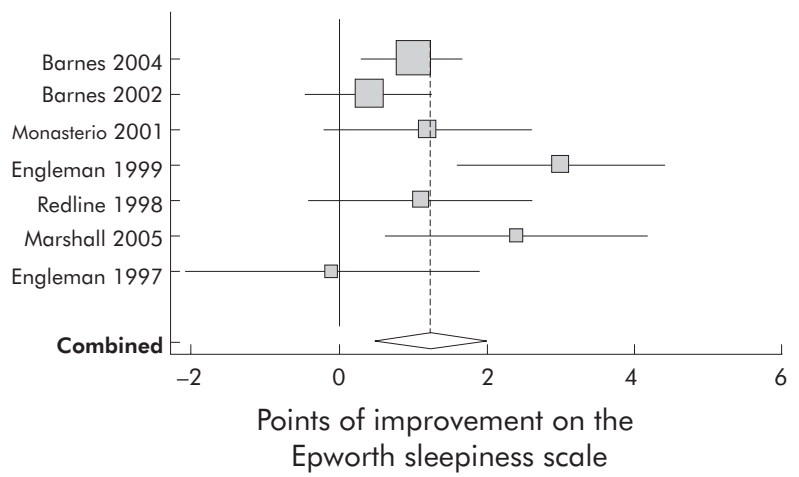

Figure 2 Forest plot indicating that Epworth sleepiness scores were significantly improved by CPAP treatment. First author and year of publication of source trial are listed on the vertical axis. Horizontal lines represent $95 \%$ confidence intervals from each indicated study for the effects of CPAP after adjustment for control. Elongated diamonds indicate the mean (apex of diamond) and $95 \%$ confidence intervals for the pooled estimate of the effect. The size of the shaded boxes represents the weight given to that study. Larger boxes are studies that have given more precise estimates and they tend to be larger.

studies measured MWT at baseline and were able to quantify the placebo effect. Barnes et al ${ }^{15}$ found a significant 2.7 minute worsening on placebo and Marshall et a ${ }^{16}$ found a non-significant 3.1 minute worsening while on placebo. These worsening sleep latencies contributed to the net effect of CPAP shown in fig 3.

The MSLT was used in four studies and worsened by a non-significant 0.2 minutes $(95 \% \mathrm{CI}-1.0$ to $0.6, \mathrm{p}=0.74$ ) with CPAP treatment after controlling for placebo effects using both fixed and random effects methods (fig 4). The effects on MSLT were not heterogeneous $(\mathrm{Q}=1.2, \mathrm{df}=3$, $\mathrm{p}=0.74)$. A comparison analysis of MSLT and MWT latencies showed that the difference in sensitivities of -0.2 minutes and 2.1 minutes, respectively, was significant (MannWhitney U test, $\mathrm{Z}=2.1, \mathrm{p}=0.03$ ).

No single trial, when removed, significantly affected the overall estimate of effects. No evidence of bias was observed

Table 2 Characteristics of patients in studies included in the meta-analysis

\begin{tabular}{|c|c|c|c|c|c|c|c|}
\hline Reference & $\begin{array}{l}\text { Mean age } \\
\text { (years) }\end{array}$ & $\begin{array}{l}\text { Sex ratio } \\
\text { (no of men) }\end{array}$ & $\begin{array}{l}\text { Mean BMI } \\
\left(\mathrm{kg} / \mathrm{m}^{2}\right)\end{array}$ & $\begin{array}{l}\text { CPAP use } \\
\text { (h/night) }\end{array}$ & $\begin{array}{l}\text { Baseline } \\
\text { ESS }\end{array}$ & $\begin{array}{l}\text { Baseline MWT } \\
\text { (min) }\end{array}$ & $\begin{array}{l}\text { Baseline MSLT } \\
\text { (min) }\end{array}$ \\
\hline Engleman $^{10}$ & 52 & $75 \%(12)$ & 30 & 2.8 & 14 & & 10 \\
\hline Redline $^{12}$ & 48 & $52 \%(50)$ & 33 & 3.1 & 10 & & 10 \\
\hline Engleman ${ }^{9}$ & 44 & $61 \%(21)$ & 30 & 2.8 & 13 & NB & \\
\hline Monasterio" & 54 & $86 \%(108)$ & 29 & 4.8 & 12 & & 10.5 \\
\hline Barnes $^{14}$ & 45 & $83 \%(35)$ & 30 & 3.5 & 11 & & 12.5 \\
\hline Barnes $^{15}$ & 46 & $79 \%(63)$ & 31 & 3.6 & 10.2 & 30.7 & \\
\hline Marshall $^{16}$ & 51 & $76 \%(22)$ & 31 & 4.9 & 12.5 & 20.9 & \\
\hline
\end{tabular}

NB, no baseline measured; BMI, body mass index; CPAP, continuous positive airway pressure; ESS, Epworth Sleepiness Scale; MSLT, Multiple Sleep Latency Test; MWT, Maintenance of Wakefulness Test. 


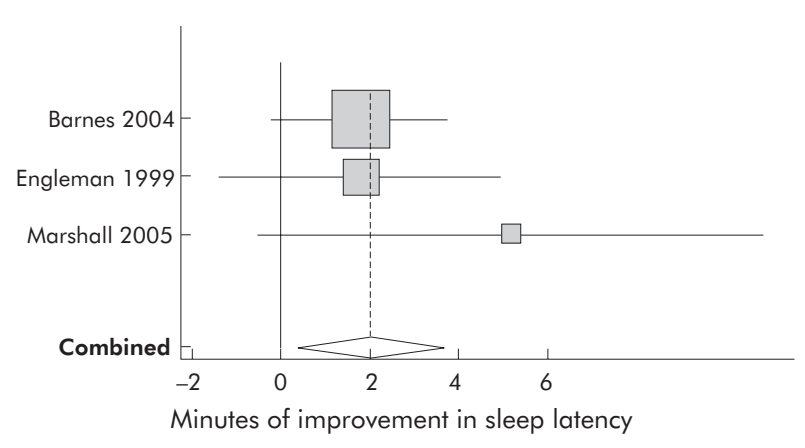

Figure 3 Forest plot indicating that Maintenance of Wakefulness Test (MWT) sleep latencies were significantly improved by CPAP therapy. First author and year of publication of source trial are listed on the vertical axis. Horizontal lines represent $95 \%$ confidence intervals from each indicated study for the effects of CPAP after adjustment for control. Elongated diamonds indicate the mean (apex of diamond) and 95\% confidence intervals for the pooled estimate of the effect.

using funnel plots or either Begg's or Egger's tests for publication bias with any of the three sleepiness measures. However, these tests for bias need to be interpreted with caution due to the combination of a few trials involving relatively small numbers of patients.

ESS scores improved by 1.2 points compared with an estimated background standard deviation of 4.5 points, ${ }^{19}$ giving an effect size of 0.27 . MWT scores improved by 2.1 minutes compared with an estimated background standard deviation of 10.2 minutes, ${ }^{19}$ giving an effect size of 0.21 . These effects are small in magnitude. ${ }^{18}$

\section{DISCUSSION}

This meta-analysis has shown that CPAP significantly reduces subjective daytime sleepiness, measured by the ESS, by 1.2 points (95\% CI 0.5 to 1.9 , effect size 0.27 ) and improves objective daytime wakefulness, measured by the MWT, by 2.1 minutes ( $95 \%$ CI 0.5 to 3.7 , effect size 0.21 ) in patients with mild to moderate OSAS. There is insufficient evidence to claim that CPAP decreases objective daytime sleepiness, as measured by the MSLT, in these patients, as sleep latencies worsened by a statistically non-significant mean of 0.2 minutes ( $95 \%$ CI -1.0 to 0.6 ).

The estimated effect on MWT latencies should to be interpreted with caution. Both trials ${ }^{15}{ }^{16}$ that reported placebo effects noted worsening on the placebo arm which is similar or larger in magnitude to the benefit of CPAP estimated here by meta-analysis. This unexpected pattern might indicate a real effect where wakefulness is becoming subtly more difficult to maintain in these patients about the time they are invited to participate in a clinical trial. It could also be evidence of a slight test-retest worsening of MWT latencies in these crossover trials. The effect size estimated by the present meta-analysis might not therefore be an accurate indication of the smaller effect that would be seen in clinical practice due to the lack of placebo adjustment. This might also be an indication that CPAP halts a decline in wakefulness that might be occurring in these patients.

The observed heterogeneity of the ESS was associated with the dropout rate, trials with low dropout rates showing significantly larger improvements. However, this might be a chance finding as it is in the opposite direction to that normally expected. Improvements in ESS scores were not related to mean compliance with CPAP or baseline disease severity (ESS or AHI), a series of potentially important mediators. Neither the MSLT nor the MWT was observed to have significant heterogeneity. Sensitivity analyses of all three outcome measures did not indicate any studies which

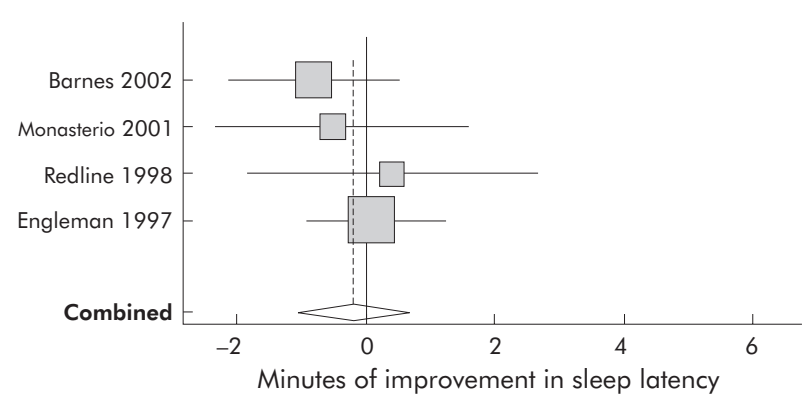

Figure 4 Forest plot indicating that Multiple Sleep Latency Test (MSLT) sleep latencies were not significantly improved by CPAP therapy. First author and year of publication of source trial are listed on the vertical axis. Horizontal lines represent $95 \%$ confidence intervals from each indicated study for the effects of CPAP after adjustment for control. Elongated diamonds indicate the mean (apex of diamond) and 95\% confidence intervals for the pooled estimate of the effect.

had undue influence upon the final estimated effects of CPAP. Funnel plots and associated statistics offered no evidence that the effects observed were due to selective publication, but this was to be expected given the relatively small size of these trials. While the tests for publication bias do not show statistical significance, it is always possible that a number of unpublished negative trials exist.

The combination of parallel and crossover trials into a single meta-analysis is not regarded as statistically orthodox because the estimates of standard error are not calculated in the same manner. However, when these study designs were separated, both analyses agreed that CPAP has a small but significant effect on subjective sleepiness that is also highly comparable to the analysis shown in fig 2. Neither type of study indicated any change in MSLT latencies, and this is also highly comparable to the effects summarised in fig 4 . Despite this agreement, our combination of these studies into single meta-analyses remains a potential study weakness.

These results extend the recent meta-analysis by Patel and colleagues $^{6}$ by meta-analysing not only improvements in the ESS score, but also MSLT and MWT improvements in patients with mild to moderate OSAS. Patel et al found that the mean benefit to subjective sleepiness in patients with mild to moderate OSAS was a non-significant 1.1 points in the ESS score compared with a significant 4.75 points for patients with largely severe OSAS. The finding here that subjective sleepiness improved by 1.2 points is almost identical in magnitude, but was statistically significant due to the additional power from the three most recent studies. The applicability of the findings here is also strengthened by the exclusion from the analyses of the study by Barbe and colleagues $^{13}$ which used CPAP to treat people with severe sleep disordered breathing but no abnormal daytime sleepiness. The study was not relevant for deciding whether to treat a group of patients with mild to moderate sleep disordered breathing and significant daytime sleepiness. In the studies analysed by Patel et al MWT latency improved by a mean of 3 minutes. Our finding of a 2.1 minute improvement in MWT latencies in a group of studies restricted to patients with mild to moderate OSAS is in accordance with expectations, given that the findings of Patel et al included patients with the full spectrum of OSAS severity (including mild to moderate). It is notable that the trials at the severe end of the disease spectrum analysed by Patel et al were of similar duration and design to the trials of mild to moderate OSAS analysed here. The greater effects seen in trials of severe OSAS are therefore not due to longer treatment durations. The paucity of effect is also not due to the poor action of the treatment as it has long been established that CPAP abolishes sleep disordered breathing while the patient is wearing the device correctly. ${ }^{5}$ 
While Patel and colleagues ${ }^{6}$ did not find significant differences between MSLT and MWT treatment sensitivities, we have from the outset assumed that these metrics should not be combined as they measure different domains. MSLT and MWT latencies in other studies are not well correlated, are differentially sensitive to CPAP treatment, and probably measure different abilities. ${ }^{27-29}$ Separating the ability to fall asleep from the ability to sustain wakefulness also seems to be supported by findings from the literature indicating that these two phenomena arise from separate but interacting neurological processes. ${ }^{30}$ The $95 \%$ confidence intervals from the meta-analyses show that, while the MSLT scores are not significantly affected by CPAP treatment, the MWT scores are significantly improved (figs 2 and 3). Furthermore, these latencies are significantly different when combined in the same meta-analysis. We therefore conclude that MSLT and MWT latencies should not be combined, despite being measured in compatible units, because the two measurements are differentially sensitive to CPAP treatment and probably measure different aspects of increased sleepiness, at least in the specific patient group included here.

The improvements in sleepiness in this group are statistically significant, but it is not clear from the present analyses whether the benefit accrues equally to those across the mild to moderate spectrum or whether there exists a mean severity point at which CPAP therapy is more of a hindrance to good sleep than the sleep disordered breathing it treats. It is also unclear whether the expense associated with ongoing CPAP treatment is cost effective in terms of the effects on daytime sleepiness. Cost effectiveness is an important consideration because the effect sizes are very small and close to being insignificant $(<0.20) \cdot{ }^{18}$ CPAP might have an expanded role in treating mild to moderate OSAS if it can be shown in randomised controlled trials to be a cost effective treatment for reducing the risk of cardiovascular disease.

In conclusion, CPAP significantly improves subjective sleepiness and objective wakefulness in patients with mild to moderate OSA; it does not improve objective sleepiness. However, the effect sizes of these adjustments are very small and may not be clinically relevant.

\section{Authors' affiliations}

N S Marshall, N Travier, P H Gander, Sleep/Wake Research Centre, Massey University, New Zealand

M Barnes, R J Pierce, Institute for Sleep and Breathing, Austin Health, Heidelberg, Victoria, Australia

N Travier, Centre for Public Health Research, Massey University, New Zealand

A J Campbell, A M Neill, WellSleep, Department of Medicine, Wellington School of Medicine, University of Otago, New Zealand R D McEvoy, Adelaide Institute for Sleep Health, Repatriation General Hospital, Daw Park, South Australia, Australia

This project was supported by the funding of a PhD stipend to NSM by Massey University and the Sleep Wake Research Centre.

Competing interests: none.

\section{REFERENCES}

1 Young T, Peppard PE, Gottlieb DJ. Epidemiology of obstructive sleep apnea: a population health perspective. Am J Respir Crit Care Med 2002; 165:1217-39.
2 Johns MW. A new method for measuring daytime sleepiness: the Epworth sleepiness scale. Sleep 1991;14:540-5.

3 Carskadon MA, Dement WD, Mitler MM, et al. Guidelines for the multiple sleep latency test (MSLT): a standard measure of sleepiness. Sleep 1986;9:519-24.

4 Mitler MM, Gujavarty KS, Browman CP. Maintenance of wakefulness test: a polysomnographic technique for evaluating treatment efficacy in patients with excessive somnolence. Electroencephalogr Clin Neurophysiol 1982:53:658-61.

5 Sullivan CE, Issa FG, Bethon-Jones $M$, et al. Reversal of obstructive sleep apnoea by continuous positive airway pressure applied through the nares. Lancet $1981 ; 1: 862-5$

6 Patel S, White D, Malhotra A, et al. Continuous positive airway pressure therapy for treating sleepiness in a diverse population with obstructive sleep apnea: results of a meta-analysis. Arch Intern Med 2003;163:565-71.

7 Young T, Palta M, Dempsey J, et al. The occurrence of sleep disordered breathing among middle aged adults. N Engl J Med 1993;328:1230-5.

8 American Academy of Sleep Medicine Taskforce. Sleep-related breathing disorders in adults: recommendations for syndrome definition and measurement techniques in clinical research. Sleep 1999;22:667-89.

9 Engleman H, Kingshott R, Wraith P, et al. Randomised placebo controlled crossover trial of continuous positive airway pressure for mild sleep apnea/ hypopnea syndrome. Am J Respir Crit Care Med 1999:159:461-7.

10 Engleman H, Martin S, Deary l, et al. Effect of CPAP therapy on daytime function in patients with mild sleep apnoea/hypopnoea syndrome. Thorax 1997; 52:114-9.

11 Monasterio C, Vidal S, Duran J, et al. Effectiveness of continuous positive airway pressure in mild sleep apnea-hypopnea syndrome. Am J Respir Crit Care Med 2001;164:939-43.

12 Redline S, Adams N, Strauss ME, et al. Improvement of mild sleep disordered breathing with CPAP compared with conservative therapy. Am J Respir Crit Care Med 1998;157:858-65.

13 Barbe F, Mayoralas L, Duran J, et al. Treatment with continuous positive airway pressure is not effective in patients with sleep apnea but no daytime sleepiness. Ann Intern Med 2001;134:1015-67.

14 Barnes M, Houston D, Worsnop C, et al. A randomized controlled trial continuous positive airway pressure in mild obstructive apnea. Am J Respir Crit Care Med 2002; 165:773-80.

15 Barnes M, McEvoy RD, Banks S, et al. Efficacy of positive airway pressure and oral appliance in mild to moderate obstructive sleep apnea. Am J Respir Crit Care Med 2004; 170:656-64.

16 Marshall NS, Neill AM, Campbell AJ, et al. Randomised controlled crossover trial of humidified continuous positive airway pressure in mild obstructive sleep apnoea. Thorax 2005;60:427-32.

17 Jadad A. Randomised controlled trials. London: BMJ Books, 1998:123.

18 Kazis L, Anderson J, Meenan R. Effect sizes for interpreting changes in health status. Med Care 1989;27(3 Suppl):S178-89.

19 Banks S, Barnes M, Tarquinio N, et al. Factors associated with maintenance of wakefulness test mean sleep latency in patients with mild to moderate obstructive sleep apnoea and normal subjects. J Sleep Res 2004;13:71-8.

20 Bao X, Nelesen R, Loredo J, et al. Blood pressure variability in obstructive sleep apnea: role of sympathetic nervous activity and effect of continuous positive airway pressure. Blood Press Monit 2002;7:301-7.

21 Profant J, Ancoli-Israel S, Dimsdale JE. A randomized, controlled trial of 1 week of continuous positive airway pressure treatment on quality of life. Heart Lung 2003;32:52-8.

22 Ziegler M, Mills P, Loredo J, et al. Effect of continuous positive airway pressure and placebo treatment on sympathetic nervous activity in patients with obstructive sleep apnea. Chest 2001;120:887-93.

23 Bardwell W, Ancoli-Israel S, Berry C, et al. Neuropsychological effects of one week continuous positive airway pressure treatment in patients with obstructive sleep apnea: a placebo controlled study. Psychosom Med $2001 ; 63: 579-84$.

24 Dimsdale J, Loredo J, Profant J. Effect of continuous positive airway pressure on blood pressure: a placebo trial. Hypertension 2000;35:144-52.

25 Yu B-H, Ancoli-Israel S, Dimsdale JE. Effect of CPAP treatment on mood states in patients with sleep apnea. J Psychiatr Res 1999;33:427-32.

26 Jokic R, Klimaszewski A, Crossley $M$, et al. Positional treatment vs continuous positive airway pressure in patients with positional obstructive sleep apnea syndrome. Chest 1999;115:771-81.

27 Sangal R, Thomas L, Mitler MM. Maintenance of wakefulness test and multiple sleep latency test: measurement of different abilities in patients with sleep disorders. Clin Neurophysiol 1992;110:2131-5.

28 Sangal R, Thomas L, Mitler MM. Disorders of excessive sleepiness: treatment improves ability to stay awake but does not reduce sleepiness. Chest 1992; 102:699-703.

29 Kingshott RN, Engleman HM, Deary IJ, et al. Does arousal frequency predict daytime function? Eur Respir J 1998;12:1264-70.

30 Espana R, Scammell T. Sleep neurobiology for the clinician. Sleep 2004;27:811-20. 